\title{
Biallelic pathogenic variants in the lanosterol synthase gene LSS involved in the cholesterol biosynthesis cause alopecia with intellectual disability, a rare recessive neuroectodermal syndrome
}

\author{
Thomas Besnard, $\mathrm{PhD}^{1,2}$, Natacha Sloboda, $\mathrm{PhD}^{3}$, Alice Goldenberg, $\mathrm{MD}^{4}$, \\ Sébastien Küry, DVM, PhD ${ }^{1,2}$, Benjamin Cogné, PharmD ${ }^{1,2}$, Flora Breheret, BSc ${ }^{1}$, Eva Trochu, BSc ${ }^{1}$, \\ Solène Conrad, $\mathrm{BSC}^{1}$, Marie Vincent, $\mathrm{MD}^{1,2}$, Wallid Deb, $\mathrm{BSC}^{1,2}$, Xavier Balguerie, $\mathrm{MD}^{5}$, \\ Sébastien Barbarot, MD, $\mathrm{PhD}^{6}$, Geneviève Baujat, MD 7 , Tawfeg Ben-Omran, MD, FRCPC 8 , \\ Anne-Claire Bursztejn, $\mathrm{MD}^{9}$, Virginie Carmignac, MD ${ }^{10,11}$, Alexandre N. Datta, $\mathrm{MD}^{12}$, \\ Aline Delignières, $\mathrm{MD}^{13}$, Laurence Faivre, $\mathrm{MD}, \mathrm{PhD}^{10,11}$, Betty Gardie, $\mathrm{PhD}^{2,14}$, \\ Jean-Louis Guéant, MD, PhD ${ }^{3}$, Paul Kuentz, MD, PhD ${ }^{10,11}$, Marion Lenglet, MSc ${ }^{2,14}$, \\ Marie-Cécile Nassogne, MD, PhD ${ }^{15}$, Vincent Ramaekers, MD, PhD ${ }^{16}$, Rhonda E. Schnur, MD ${ }^{17}$, \\ Yue Si, MD, PhD ${ }^{17}$, Erin Torti, MS ${ }^{17}$, Julien Thevenon, MD, PhD ${ }^{18}$, Pierre Vabres, MD, PhD ${ }^{10,11}$, \\ Lionel Van Maldergem, MD, PhD ${ }^{19,20}$, Dorothea Wand, $\mathrm{MD}^{21}$, Arnaud Wiedemann, $\mathrm{MD}^{3}$, \\ Bertrand Cariou, $\mathrm{MD}, \mathrm{PhD}^{2}$, Richard Redon, $\mathrm{PhD}^{2}$, Antonin Lamazière, $\mathrm{MD}, \mathrm{PhD}^{22}$, \\ Stéphane Bézieau, PharmD, PhD ${ }^{1,2}$, Francois Feillet, MD, PhD ${ }^{3}$ and Bertrand Isidor, MD, $\mathrm{PhD}^{1,2}$
}

Purpose: Lanosterol synthase (LSS) gene was initially described in families with extensive congenital cataracts. Recently, a study has highlighted LSS associated with hypotrichosis simplex. We expanded the phenotypic spectrum of LSS to a recessive neuroectodermal syndrome formerly named alopecia with mental retardation (APMR) syndrome. It is a rare autosomal recessive condition characterized by hypotrichosis and intellectual disability (ID) or developmental delay (DD), frequently associated with earlyonset epilepsy and other dermatological features.

Methods: Through a multicenter international collaborative study, we identified LSS pathogenic variants in APMR individuals either by exome sequencing or LSS Sanger sequencing. Splicing defects were assessed by transcript analysis and minigene assay.

Results: We reported ten APMR individuals from six unrelated families with biallelic variants in LSS. We additionally identified one affected individual with a single rare variant in LSS and an allelic imbalance suggesting a second event. Among the identified variants, two were truncating, seven were missense, and two were splicing variants. Quantification of cholesterol and its precursors did not reveal noticeable imbalance.

Conclusion: In the cholesterol biosynthesis pathway, lanosterol synthase leads to the cyclization of (S)-2,3-oxidosqualene into lanosterol. Our data suggest LSS as a major gene causing a rare recessive neuroectodermal syndrome.

Genetics in Medicine (2019) 21:2025-2035; https://doi.org/10.1038/s41436019-0445-x

Keywords: LSS; intellectual disability; cholesterol pathway; early-onset epileptic encephalopathy; alopecia

\section{INTRODUCTION}

Cholesterol and its precursors are essential components in the structure and function of the human central nervous system (CNS) and peripheral nervous system, as well as in embryonic and postnatal development. Cholesterol synthesis in the developing CNS is relatively high, and declines to a very low level in the adult state. ${ }^{1}$ The brain contains about $23 \%$ of the whole body's cholesterol. ${ }^{2,3}$ Myelin sheaths account for approximately $70 \%$ of brain cholesterol and the remaining $30 \%$ is included in the plasma membranes of astrocytes and neurons. ${ }^{1}$ Cholesterol may contribute to maintaining the complex morphology and synaptic transmission of astrocytes and neurons. ${ }^{3}$ When cholesterol homeostasis is altered, it contributes to human disease, from common disorders like familial hypercholesterolemia and related cardiovascular diseases (MIM 143890) to rare genetic syndromes, including fetal developmental disorders with autosomal recessive inheritance such as Smith-Lemli-Opitz syndrome (SLOS; MIM 270400), lathosterolosis (MIM 607330), desmosterolosis (MIM 602398),

Correspondence: Thomas Besnard (thomas.besnard@chu-nantes.fr) or Bertrand Isidor (bertrand.isidor@chu-nantes.fr). ”Affiliations are listed at the end of the paper. 
and sterol-C-4 methyloxidase-like deficiency (SC4MOL; MIM 616834). Sterol disorders with X-linked dominant inheritance include X-linked dominant chondrodysplasia punctata (CDPX2; MIM 302960) and congenital hemidysplasia with ichthyosiform erythroderma and limb defects syndrome ${ }^{4}$ (CHILD; MIM 308050) (Figure Supp 1). The pathological processes could be the consequence of cholesterol deficiency, the accumulation of sterol precursors, ${ }^{4}$ or the dysregulation of Sonic Hedgehog ( $\mathrm{SHH}$ ) signaling during fetal life. ${ }^{5-7}$ Recently, a study highlighted alterations of the squalene synthase gene (FDFT1) as responsible for a monogenic autosomal recessive disorder (squalene synthase deficiency [SQSD]; MIM 618156) with profound developmental delay, brain abnormalities, 2-3 syndactyly of the toes, and facial dysmorphism, similar to Smith-Lemli-Opitz syndrome. $^{8}$ Biallelic missense variants in the lanosterol synthase (LSS) gene ${ }^{9,10}$ were also associated with a phenotype that included congenital cataracts in three families. In one individual, ${ }^{10}$ the affected child also had congenital alopecia and small penis. Recently, LSS was also associated with hypotrichosis simplex in three unrelated families but intellectual disability in siblings from one of these families was considered as coincidental by the authors. ${ }^{11}$

Cellular cholesterol synthesis is a complex process starting with the conversion of acetyl coenzyme A (acetyl-CoA) in $\beta$ Hydroxy $\beta$-methylglutaryl-CoA (HMG-CoA) by HMG-CoA synthase. $^{3,4}$ The first sterol product of the pathway is lanosterol, synthesized by the conversion of (S)-2,3oxidosqualene by the lanosterol synthase (LSS) enzyme. ${ }^{12}$ Lanosterol is the common substrate of two subsequent pathways of the biosynthesis of cholesterol: the Bloch and the Kandutsch-Russell pathways ${ }^{4}$ (Figure Supp 1).

Alopecia and mental retardation (APMR) syndrome (MIM 203650) is a rare disorder with autosomal recessive transmission. Only a few families have been identified and affected individuals are characterized by the absence of scalp hair, the absence of eyebrows and eyelashes, and variable intellectual disability. ${ }^{13}$ In 1992, a subdivision into three groups of APMR syndrome was proposed depending on the association with microcephaly and epilepsy: ${ }^{14,15}$ (1) APMR with microcephaly without epilepsy, (2) APMR without microcephaly, and (3) APMR with microcephaly and epilepsy. To date, variants in the gene coding for the a-2HS-glycoprotein (AHSG) have been reported in one consanguineous family, suggesting $A H S G$ as a potential cause of APMR. ${ }^{13}$ Moreover, three loci have been associated with APMR by linkage analyses: APMR1 (MIM 203650), APMR2 (MIM 610422), and APMR3 (MIM 613930) have been mapped to chromosomes 3q26.33- q27.3 (ref. ${ }^{16}$ ), 3q26.2-q26.31 (ref. ${ }^{17}$ ), and 18q11.2-q12.2 (ref. ${ }^{18}$ ) respectively. Another study reported additional loci (1p31.1-p22.3, $8 \mathrm{p} 23.1,8 \mathrm{p} 22-\mathrm{p} 21.3$, and $14 \mathrm{q} 24.3-\mathrm{q} 31.3$ ) likely to be associated with APMR syndrome. ${ }^{19}$ None of the previously described loci include the LSS gene (21q22.3).

\section{MATERIALS AND METHODS}

\section{Patients}

Here, we report 11 individuals from seven unrelated families affected with alopecia, scaly skin, other ectodermal abnormalities, variable magnetic resonance image (MRI) abnormalities and neurological symptoms. The mode of inheritance for the disorder is most likely autosomal recessive (Fig. 1, Table 1). Clinical description of each individual is available in the Supplementary data. Subjects were enrolled from five investigating centers: Nantes, Nancy, and Rouen in France; Basel in Switzerland; Brussels in Belgium; and Doha in Qatar. According to the pedigrees (Fig. 1, Table 1) and previous studies on APMR, an autosomal recessive inheritance pattern is suspected.

Written informed consent was obtained for all affected individuals and relatives. The study was approved by the CHU de Nantes-ethics committee (Comité consultatif sur le traitement de l'information en matière de recherche; number CCTIRS: 14.556). Additional written consent to use photographs in this publication was obtained for individuals from families F1, F2, F3, F5, and F7.

Exome sequencing (ES) was performed for 5 of the 11 individuals in three families: F1 (F1-II-2 and F1-II-3), F2 (F2-II-1), and F3 (F3-II-1 and F3-II-3) (Fig. 1). Rare or never identified LSS biallelic variants (gnomAD frequency $<2.894^{\star} 10^{-5}$ ) were identified in the five individuals (Fig. 3). Individual F7-II-1 was identified with LSS-related disease via GeneMatcher. ${ }^{20}$ Assuming that likely pathogenic variants in LSS might account for the disease in other individuals with unresolved APMR phenotype, we tested three additional families (families F4, F5, and F6) by Sanger sequencing of the 22 exons and intronic flanking sequences of LSS (GeneBank: NM_002340.5; NP_002331.3).

\section{Exome sequencing strategy}

Exome sequencing (ES) for families F1 and F2 was performed with SeqCap EZ MedExome (Roche) following recommended procedures and samples were sequenced on NextSeq500. Paired-end reads of $75 \mathrm{bp}$ were obtained. Quality of Fastq files was confirmed by Fastqc (v0.11.3), adapters were removed by cutadapt (v1.8.1), and reads were processed following a pipeline according to Genome Analysis Toolkit (GATK)'s best practices (v3.4). Briefly, Fastq files were aligned on human genome hg19 by BWA-MEM (v0.7.3), BAM files were sorted, and duplicate reads were filtered out using Picard MarkDuplicates (v1.119). Alignment files were then realigned and base quality recalibrated. Variant calling was performed using HapolypeCaller (GATK) in gVCF mode and gVCF was genotyped together with 30 other ES gVCFs. Variants were annotated using ANNOVAR. Variants with a frequency over $0.5 \%$ in 1000 Genomes, Exome Variant Server (EVS), and ExAC and with a frequency over $1 \%$ in a local database were filtered out. ES strategies followed by each participating center are available upon request. 

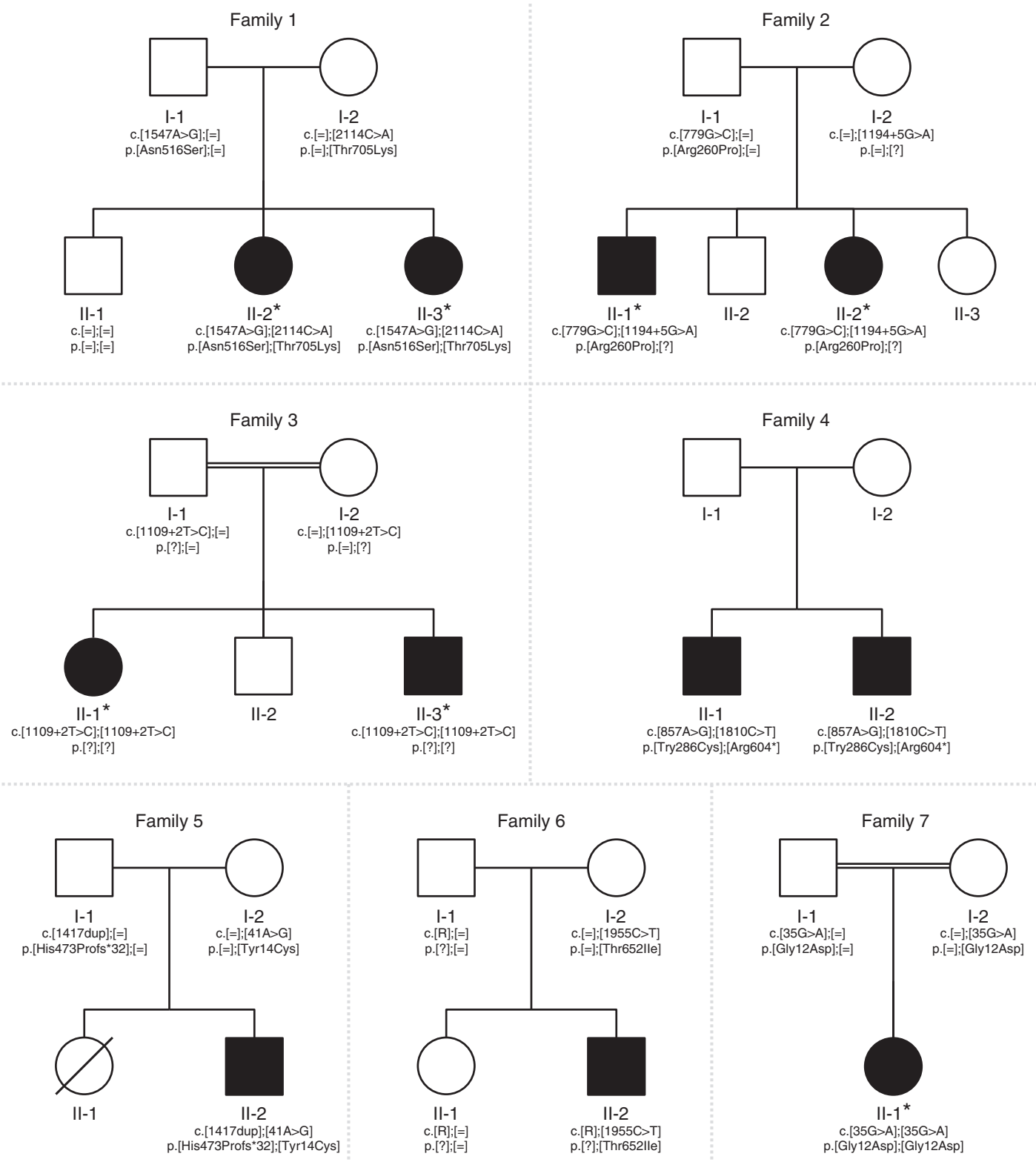

Fig. 1 Pedigrees of the six families affected by LSS pathogenic variants. Asterisks (*) mean that individuals were exome sequenced. The identified LSS variants were Sanger sequenced in all available relatives. mut/mut refers to individuals with homozygous or compound heterozygous $L S S$ variants, mut/refers to heterozygous carriers of $L S S$ pathogenic variants, and -/- indicates individuals for whom no LSS pathogenic variation was highlighted. [R] indicates a LSS not currently identified variant is suspected on the allele leading to a decrease of the transcript quantity.

\section{Transcript and splicing reporter minigenes analysis}

When biological material was available, transcript study was performed. Biological material could be blood samples from PAXgene blood RNA tubes, lymphocytes from blood samples, or fibroblasts, depending on the families. Total RNA was isolated with the NucleoSpin RNA Plus Kit (Macherey-Nagel) according to the manufacturer's instructions. The concentration of total RNA was measured on a NanoDrop spectrophotometer (NanoDrop Technologies). Reverse transcription was performed with the ProtoScript II First Strand cDNA Synthesis Kit (New England Biolabs). Polymerase chain reaction (PCR) was performed on complementary DNA (cDNA) with the HotStarTaq DNA Polymerase (Qiagen) or the Platinum DNA Polymerase (Thermo Fisher Scientific) according to the PCR amplicons (PCR conditions are available upon request).

To evaluate the impact on splicing of c.1109+2T>C and c.1194+5G $>$ A variants, we performed splicing minigene reporter assays using the pCAS2 vector based on a previously described protocol. ${ }^{21}$ This functional assay is based on the comparative analysis of the splicing pattern of wild-type and mutant. 


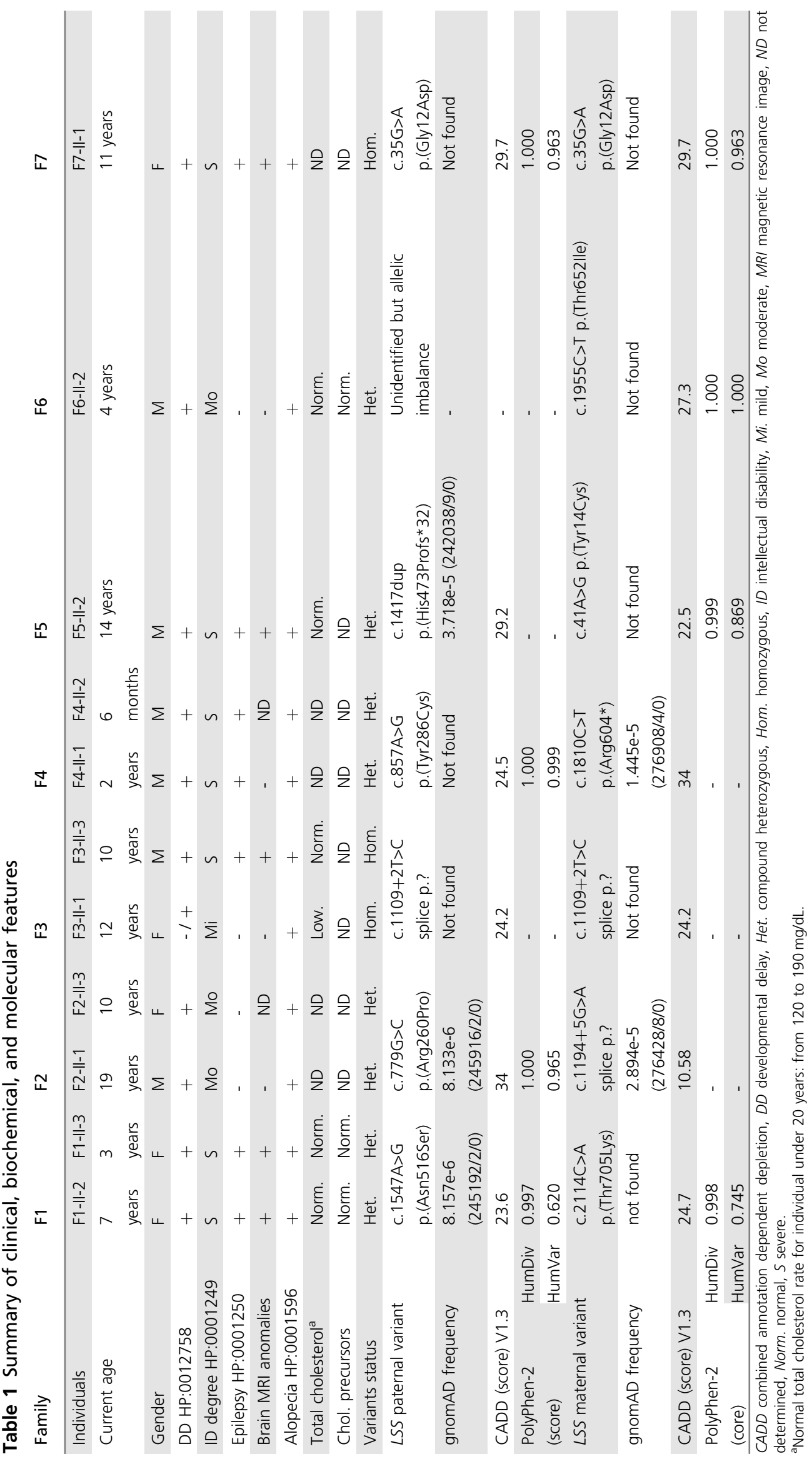




\section{Biochemical analyses}

Cholesterol precuror, oxysterol, and cholesterol analysis followed the "gold standard" method ${ }^{22}$. Briefly, a mix of internal standards (epicoprostanol, 2H7-7-lathosterol, 2H6desmosterol, 2H6-lanosterol, and 2H6-24[S]-hydroxycholesterol [Avanti Polar Lipids]) was added to $0.2 \mathrm{ml}$ of plasma samples. Extraction of the lipid fraction was achieved with $5 \mathrm{ml} \mathrm{MTBE} / \mathrm{MeOH}(10 / 3)$ and $2 \mathrm{ml} \mathrm{NaCl}$ solution (0.9\%). Alkaline hydrolysis was then performed under Ar using $0.5 \mathrm{M}$ methanolic $\mathrm{KOH}$ for 15 minutes at $65^{\circ} \mathrm{C}$. Next, $1 \mathrm{ml}$ of $\mathrm{BF} 3$ was added and the solution was kept at $65^{\circ} \mathrm{C}$ for 15 minutes. After cooling the solution, $5 \mathrm{ml}$ of cyclohexane was added to collect the sterol phase. The upper phase was collected and dried under a stream of nitrogen. Sterols and oxysterols were silylated with Regisil $^{\circledR}+10 \%$ TMCS (bis[trimethylsilyl] trifluoroacetamide $+10 \%$ trimethylchlorosilane [Regis Technologies]) as described previously ${ }^{23}$. The trimethylsilylether derivatives of sterols and oxysterols were separated by gas chromatography (Hewlett Packard 6890 series) in a medium polarity capillary column RTX-65 (65\% diphenyl $35 \%$ dimethyl polysiloxane, length $30 \mathrm{~m}$, diameter $0.32 \mathrm{~mm}$, film thickness $0.25 \mathrm{~mm}$; Restesk). The mass spectrometer (Agilent 5975 inert XL) in series with the gas chromatography was set up for detection of positive ions. Ions were produced in the electron impact mode at $70 \mathrm{eV}$. They were identified by fragmentogram in scanning mode and quantified by selective monitoring of the specific ions after normalization and calibration with the appropriate internal and external standards.

\section{RESULTS}

The 11 individuals ( 6 males and 5 females) all presented with congenital alopecia and developmental delay (Table 1, Fig. 2). Total alopecia with sparse eyelashes and eyebrows was noted at birth in all affected individuals. Other dermatological disorders such as ichthyosis and erythroderma were found in 9/11 individuals (Supplementary Table 1). Intellectual disability was considered mild (1/11), moderate (3/11), or severe (7/11). Microcephaly was not constant (3/8). Epilepsy was also frequent (8/11). The age of onset of the epileptic disease ranged from birth to 10 weeks. Neurodevelopmental abnormalities (Supplementary Table 1) included absent or very poor speech in 9/10 individuals, hypotonia in 7/11. Cholesterol levels were normal for $4 / 5$ individuals. Brain MRI showed nonspecific abnormalities (6/9). Interestingly, four males presented with genital abnormalities.

Individuals F1-II-2 (Fig. 2a) and F1-II-3 were compound heterozygous for two missense variants: c.1547A $>\mathrm{G}$; p.(Asn516Ser) in exon 16 and c.2114C>A; p.(Thr705Lys) in exon 22 (Table 1, Figs. 1 and 3), each inherited from a parent (Figure Supp 1). The healthy brother F1-II-1 was not a carrier of either of these two variants. The c.1547A $>\mathrm{G}$ change had been reported twice (frequency: 8.157e-6) and the substitution c. $2114 \mathrm{C}>\mathrm{A}$ had never been observed previously in the gnomAD population. The substitution c. $2114 \mathrm{C}>\mathrm{T}$ had already been identified (gnomAD frequency: 2.461e-5), albeit its frequency remained consistent with postulated recessive mode of inheritance. Both of these missense variants were predicted as potentially pathogenic by bioinformatics tools, with combined annotation dependent depletion (CADD) ${ }^{24}$ scores of 23.6 and 24.7 for p.(Asn516Ser) and p.(Thr705Lys), respectively.

Individuals F2-II-1 (Fig. 2f) and F2-II-3 (Fig. 2e) were compound heterozygous for a paternally inherited missense variant c.779G $>$ C; p.(Arg260Pro) in exon 7 and a maternally inherited splice-site donor substitution c.1194+5G>A in intron 12 (Table 1, Fig. 3). The missense variant p.(Arg260Pro) was predicted to be pathogenic (CADD score of 34.0). All relevant bioinformatics programs predicted the abolition of the donor splice site by variant c. $1194+5 \mathrm{G}>\mathrm{A}$ (Fig. 4). Minigene assays confirmed the effect of the variant on mRNA splicing and showed that the variant predominantly induced in-frame skipping of exon 12 (Fig. 4).

Individuals F3-II-1 (Fig. 2b) and F3-II-3 (Fig. 2c) were homozygous for splice-site donor variant c. $1109+2 \mathrm{~T}>\mathrm{C}$ in intron 10. This variant was not found in the gnomAD database and was predicted to abolish the donor splice site. The splicing consequences of the variant were also assessed by minigene study (Fig. 4). Skipping of the exon 10 is predominant although there is also some normal splicing but weak. RT-PCR sequencing on total RNA from fibroblasts of the family F3 confirmed this result.

Individuals F4-II-1 and F4-II-2 were compound heterozygous for a missense variant c.857A $>\mathrm{G}$; p.(Tyr286Cys) in exon 8 and a nonsense variant c.1810C>T; p. (Arg604 $\left.{ }^{\star}\right)$ in exon 19 (Table 1). The c.857A $>\mathrm{G}$ variation had not been previously identified in the gnomAD database and was predicted pathogenic (CADD: 24.5). Nonsense p.(Arg604*) variant was present four times in gnomAD (frequency: 1.445e-5).

Individual F5-II-2 (Fig. 2d) was compound heterozygous for a maternally inherited missense variant c. $41 \mathrm{~A}>\mathrm{G}$; p.(Tyr14Cys) in exon 2 and the paternally inherited frameshift variant c.1417dup; p.(His473Profs $s^{\star} 32$ ) in exon 15 (Table 1). The c. $41 \mathrm{~A}>\mathrm{G}$ variation had not been previously identified in the gnomAD database and the in silico prediction tools were for a pathogenic effect (CADD: 22.5). The variant c.1417dup leading to a premature stop codon was reported five times in gnomAD (frequency: 3.718e-5).

To date, in family F6 (Fig. 2g), only one variant c.1955C>T; p.(Thr652Ile) was found in exon 20 in the affected individual F6-II-2. This maternally inherited missense variant is absent from the gnomAD database. Linkage analysis using single nucleotide polymorphisms of LSS revealed that affected individual (F6-II-2) and unaffected sister (F6-II-1) inherited the same paternal allele, but that they inherited a different allele from their mother (Figure Supp 2). However, we observed that the transcript corresponding to the paternal allele was less expressed than the one related to the alternative allele. RT-PCR from fibroblast RNA revealed an allelic imbalance noticeable in the individual F6-II-2 and the father F6-I-1 (Figure Supp 3). Fibroblast RNA from the healthy 

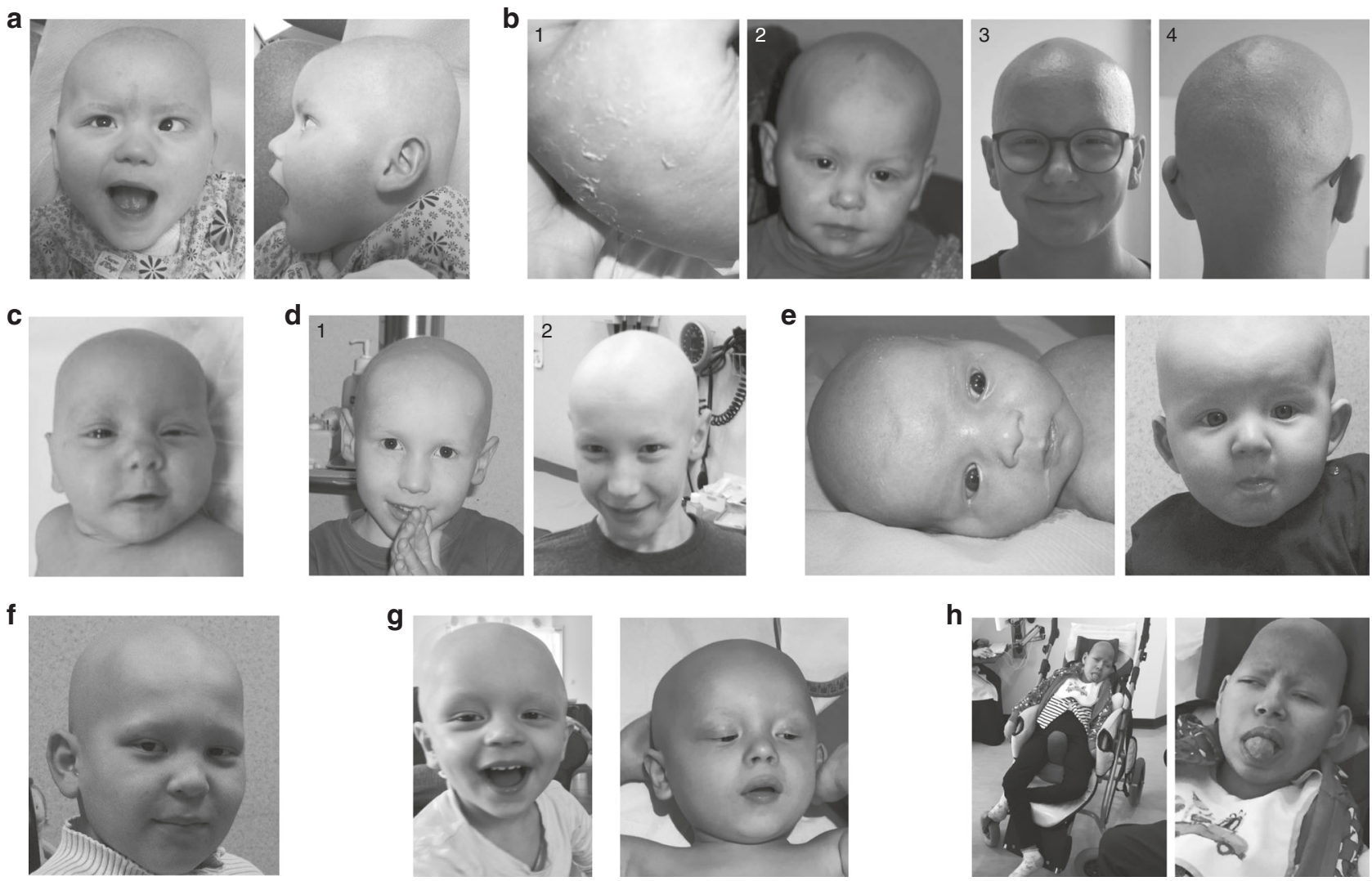

Fig. 2 Individual pictures. a Individual F1-II-2 at 19 months. b Individual F3-II-1 at birth: cutaneous desquamation (1), at 15 months (2), and at 8 years old (3 and 4). c Individual F3-II-3 at 6 months. d Individual F5-II-2 at 4 (1) and 11 years old (2). e Individual F2-II-3 at 6 months. (f) Individual F2-II-1 at 10 years old. $\mathbf{g}$ Individual F6-II-1 at 2 years old. $\mathbf{h}$ Individual F7-II-1 at 11 years old. Some common morphologic features: wide nasal base (a, b, e, $\mathbf{f}, \mathbf{g})$ prominent forehead $(\mathbf{c}, \mathbf{e}, \mathbf{f})$, and triangular face.

sister (F6-II-1) was not available. The cause of this allelic imbalance has not been clarified yet.

Individual F7-II-1 (Fig. 2h) was homozygous for the missense variant c.35A $>$ G; p.(Gly12Asp), which was predicted to be pathogenic (CADD : 29.7), was evolutionary conserved and was absent in the gnomAD database.

Eleven different variants were identified among the seven unrelated families. Seven of them were missense variants, one was nonsense, one was frameshift leading to a premature termination codon, and two were splice variants. Variants were distributed all along LSS gene. All missense variants were found to affect highly conserved amino acids (Fig. 3) and were predicted as likely pathogenic with a CADD score higher than 22.5 (Table 1). Five of the seven missense variants were not found in gnomAD and two were found at very low frequency. For all the families, heterozygous carriers were asymptomatic with normal hair and no neurological symptom. According to the Genome Aggregation Consortium (gnomAD) Browser data, only 41 loss-of-function variants in LSS were detected 123,136 exomes and 15,496 genomes present in the database ${ }^{25}$ none of them were homozygous.

To improve the characterization of the missense variants, we performed a structural analysis using 3D modeling for missense variants (Fig. 3e, f). Lanosterol synthase is an integral monotopic protein which is inserted in the membrane without spanning the lipidic bilayer. ${ }^{26,27}$ The LSS model was built from the structure inferred from X-ray crystallographic analyses (1W6K in Protein Data Bank), as previously determined. ${ }^{27}$ Missense variants identified in individuals from our cohort were located throughout the protein. 3D modeling did not reveal any clustering. Missense variants were tested into the model by using the most likely rotamer selected. We showed that they could impact the protein conformation either by either disrupting hydrogen bonds (p.[Gly12Asp] and p.[Thr652Ile]) or inducing steric hindrance (p.[Tyr14Cys], p.[Arg260Pro], p.[Tyr286Cys], p.[Asn516Ser], and p.[Thr705Lys]). Along with the high CADD scores, the absence or very low frequency in population databases and the segregation in families with the pathology, the 3D modeling provides additional supporting evidence for the potential pathogenicity of these variants.

Sterol profiling was achieved using a GC-MS approach to discriminate and quantify a maximum number of metabolites downstream in the cholesterol pathway. Plasma cholesterol levels of 6 individuals did not significantly vary from controls $(n=10)$. Circulating sterol precursor concentrations such as 8-Dehydrocholesterol and 7Dehydrocholesterol were decreased in some individuals, 
but they were not in a pathological range. The others metabolites (precursors, oxysterols and phytosterols) did not exhibit any particular abnormalities (Figure Supp 4). Specific accumulation of squalene molecular species was not detected in either individual serum or their culture fibroblast cells (data not shown).
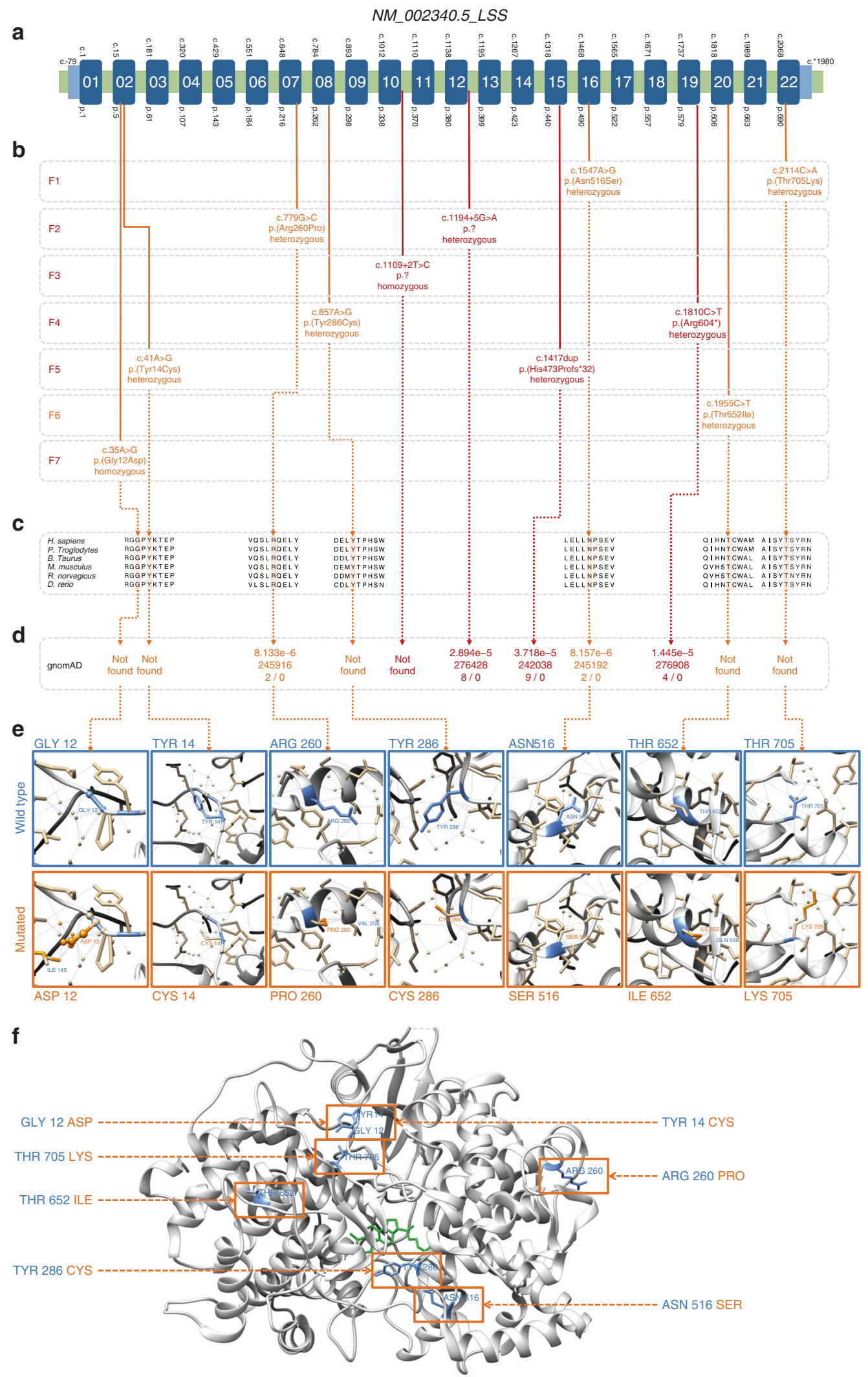
Fig. 3 Pathogenic variants identified in LSS in alopecia with mental retardation (APMR) syndrome individuals. a Schematic representation of the 22 exons of the predominant LSS transcript (NM_002340.5). b The 11 variants are represented by family. Red variants correspond to truncating and splicing variants. Orange variants are missense. c Alignment showing the conservation of amino acid residues altered by the missense variants. $\mathbf{d}$ Allele frequency when available in gnomAD database. e 3D modeling of the impact of the LSS missense variants. Blue panels represent the wild-type residue. Mutated residues were introduced by selecting the most probable rotamer and are represented by orange panels. Thin blue lines indicate the hydrogen bonds. Red lines and orange spots show the steric clashes. $\mathbf{f} 3 \mathrm{D}$ modeling of the whole LSS protein. Wild-type residues for each missense are indicated in blue and lanosterol in green. University of California-Santa Cruz (UCSC) Chimera software was used to visualize modeling and to predict consequences of the mutated residues. LSS model was built from the structure downloaded in the Protein Data Bank as entry 1W6K.

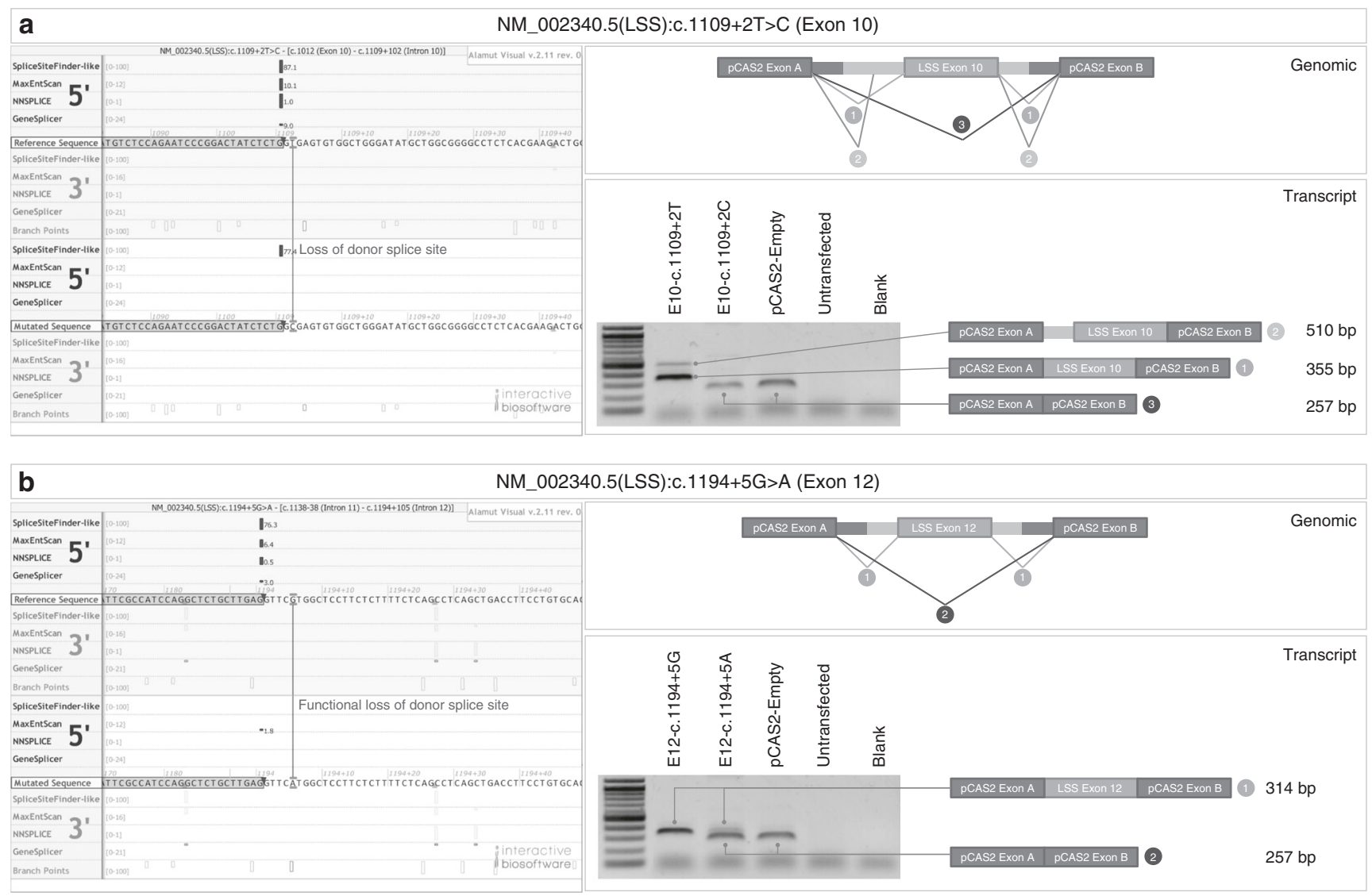

Fig. 4 Predicted effect of splice-site variants. According to prediction programs Splice Site Finder-like, MaxEntScan, NNSplice, and Gene Splicer compiled by Alamut Visual v2.11 (Interactive Biosoftware, Rouen, France), the two variants LSS c.1109+2T>C and c.1194+5G >A induce a loss of canonical donor splice sites (see left panels). The most likely predicted consequence is a skipping of out-of-frame LSS exon 10 (98 bp) for C.1109+2T>C and in-frame LSS exon 12 (57 bp) for c.1194+5G>A. Predictions about the effects of these variants were confirmed in vitro by used of a pCAS2 minigene system (see rights panels); PCR products were visualized on 1.5\% agarose gel and Sanger sequencing were performed (data not shown). a Three different transcripts were identified with minigene constructs of exon 10. Transcript 1 corresponds to the physiological splicing of the exon 10. Transcript 2 is a non physiological splicing artifact occuring only with the wild-type (WT) construct (E10-c.1109+2T). The mutant minigene construct (E10-c.1109+2T) leads mostly to the transcript 3 corresponding to the skipping of the exon 10. A remaining physiological splicing is weakly present (barely detectable on gel but present in sanger sequencing). b Minigene constructs of the exon 12 reveal two different transcripts. The WT construct (E12-c. 1194+5G) highlights the transcript 1 corresponding to the physiological splicing of the exon 12. However mutant construct (E12-c.1194+5A) presents predominantly the transcript 2 corresponding to a complete skipping of the exon 12 but also the transcript 1. In the in vitro model, the donor splice site seems not completely disturbed by the variant E12-c.1194+5.DNA variants are described according to the nomenclature established by the Human Genome Variation Society. Nomenclature HGVS V2.0 is defined according to LSS mRNA reference sequence NM_002340.5. Nucleotide numbering uses +1 as the A of the ATG translation initiation codon in the reference sequence, with the initiation codon as codon $\overline{1}$.

\section{DISCUSSION}

LSS encodes lanosterol synthase (2,3-oxidosqualene-lanosterol cyclase; OSC or LSS; EC 5.4.99.7) and maps to chromosome $21 \mathrm{q} 22.3$ (ref. ${ }^{28}$ ). Lanosterol synthase is highly conserved across species with about $83 \%, 40 \%$, and $39 \%$ conservation between human and rat, S.cerevisiae, and C. albicans, respectively. ${ }^{28}$ LSS protein plays a key role in the sterol biosynthesis pathway by catalyzing the conversion (cyclization) of (S)-2,3-oxidosqualene to lanosterol, the first sterol of the pathway. ${ }^{29}$ 
LSS was initially described as a candidate gene for holoprosencephaly because of its localization within the HPE1 locus on chromosome $21 \mathrm{q} 22.3$ (ref. ${ }^{30}$ ). LSS has also been associated with congenital cataract in rat and humans. 9,10,31 Thus, a combination of pathogenic variants in the Lss and Fdft1 genes decreases the cholesterol levels in rat lenses and has been associated with cataract formation in the Shumiya cataract rat (SCR) strain. ${ }^{31}$ Affected two families with congenital cataract were then found to have LSS homozygous missense variants: c.1741T $>$ C; p.(Trp581Arg) and c.1762G $>$ A; p.(Gly588Ser). ${ }^{9}$ More recently, a new individual affected by congenital cataract has been reported ${ }^{10}$ with compound heterozygous variants c.1025T>G; p.(Ile342Ser) and c.1887G>T; p.(Trp629Cys) in LSS. Interestingly, this child also showed alopecia and absent eyebrows, but no neurodevelopmental disorder was reported. In our cohort, none of the individual had congenital cataract.

Recently, a study has associated biallelic variants to hypotrichosis simplex in three unrelated families. ${ }^{11}$ Although in a family, both of the siblings presented intellectual disability, authors considered ID as coincidental, notably because ID was not apparent in other sibling of their study. Our data does not support this conclusion because all individuals of our study presented ID.

Although most of the individuals of the cohort showed congenital alopecia and neurodevelopmental symptoms, we observed clinical variability between families, more especially with respect to epilepsy or brain anomaly. There was also intrafamilial phenotypic variability in family 3 in which individual F3-II-1 did not show severe developmental delay as compared with her brother F3-II-3 who was very delayed. This phenotypic variability is also described in other cholesterol biosynthesis deficiency syndromes such as SLOS. ${ }^{5}$ Whereas the different LSS variants between families could explain the interfamilial phenotypic variability, the etiology of the intrafamilial variability remains unclear.

All Individuals in our cohort each carry at least one missense variant, except for Individuals F3-II-1 and F3-II-3 (family 3) carrying the homozygous LSS splice-site donor variant c. $1109+2 \mathrm{~T}>\mathrm{C}$. We assume for the missense variants, lanosterol synthase could be still synthetized but would likely have reduced activity. In family 3, skipping of exon 10 seems not to be total (Fig. 4); the remaining amount of lanosterol synthase might be sufficient to be compatible with life. None of our cases carried biallelic variants that predicted a total silencing of the protein synthesis in our cohort. It is possible that complete absence of lanosterol synthase activity would be lethal. This hypothesis is supported by the results available in the International Mouse Phenotyping Consortium website. Indeed, homozygous mice for the allele Lss ${ }^{\text {tmlb(KOMP)Wtsi }}$ demonstrate variable lethality, from embryonic day 9.5 to postnatal prior to weaning (http://www.mousephenotype. org). Interestingly, the DHCR7 and DHCR24 genes involved in the cholesterol biosynthesis pathway show the same complexity. DHCR24 encodes $3 \beta$-hydroxysterol- $\Delta 24$ reductase, an enzyme that catalyzes the conversion of desmosterol to cholesterol. A defect in this gene causes an autosomal recessive disorder, desmosterolosis. ${ }^{32,33}$ In the mouse model, all Dhcr24-/- mice died a few hours after birth. ${ }^{34}$ DHCR7 encodes $3 \beta$-hydroxysteroid- $\Delta 7$-reductase and pathogenic variants in this gene are responsible for SLOS (MIM 270400) with a frequently severe human phenotype. In Dhcr $7^{-1-}$ knockout mice, prenatal lethality was not identified but newborn mice died soon after birth. ${ }^{35}$ Similar results were shown for FDFT1, coding for the enzyme squalene synthase. Embryonic lethality in Fdft1-null mice at day 12.5 was associated with growth restriction and neurodevelopmental disorders. ${ }^{36}$ In a recent study, ${ }^{8}$ the authors postulated that the phenotypic viability in individuals with compound heterozygous variants in FDFT1 was related to a residual FDFT1 activity, depending on the nature of the pathogenic variant. ${ }^{8}$

Defects in cholesterol biosynthesis are known to be involved in several inherited human malformation syndromes. Among these syndromes, three (CHILD, CDPX2, and desmosterolosis) exhibit multiple cutaneous abnormalities, including alopecia. ${ }^{4}$ Ichthyosiform erythoderma is a feature of CHILD syndrome $^{4}$ and similar ectodermal abnormalities were frequently reported in individuals with biallelic variants in LSS. More recently, the cholesterol biosynthetic pathway was found to be impaired in the skin and hair follicles of individuals with primary cicatricial alopecia, an inflammatory hair disorder causing scarring and permanent hair loss. ${ }^{37}$ Moreover, male genitalia anomalies (4/6 in the cohort) are frequent in cholesterol biosynthesis inborn errors since fine regulation of cholesterol biosynthesis during fetal life is necessary for the development of normal genitalia. ${ }^{38-40}$

In conclusion, our study adds APMR syndrome to the list of disorders associated with alteration of genes involved in cholesterol biosynthesis. We provide evidence that APMR syndrome is a recognizable neurodevelopmental and ectodermal disorder associated with LSS biallelic pathogenic variants, which is very important for genetic counseling and would allow for the provision of antenatal diagnosis in affected families. LSS is now associated to a very wide phenotypic spectrum, from congenital cataract to APMR syndrome, including hypotrichosis simplex. Future studies will attempt to determine a more precise link between the defect in cholesterol biosynthesis and the biological basis of the neurological and dermatological symptoms found in these patients.

URLs: CADD scores (Combined Annotation Dependent Depletion): https://cadd.gs.washington.edu/ ExAC Browser: http:/exac.broadinstitute.org/ gnomAD: http://gnomad. broadinstitute.org/ GenBank: http://www.ncbi.nlm.nih.gov/ genbank/ OMIM: http://www.omim.org/ UniProt: http:// www.uniprot.org/uniprot/

\section{SUPPLEMENTARY INFORMATION}

The online version of this article (https://doi.org/10.1038/s41436019-0445-x) contains supplementary material, which is available to authorized users. 


\section{ACKNOWLEDGEMENTS}

We thank all families for participating in this study. We are also most grateful to the Rotary Club des Sables d'Olonne for support of this project.

\section{DISCLOSURE}

Y.S., R.E.S., and E.T. are employees of GeneDx, Inc., a wholly owned subsidiary of OPKO Health, Inc. The other authors declare no conflicts of interest.

Publisher's note: Springer Nature remains neutral with regard to jurisdictional claims in published maps and institutional affiliations.

\section{REFERENCES}

1. Björkhem I, Meaney S. Brain cholesterol: long secret life behind a barrier. Arterioscler Thromb Vasc Biol. 2004;24:806-815.

2. Dietschy JM, Turley SD. Thematic review series: brain Lipids. Cholesterol metabolism in the central nervous system during early development and in the mature animal. J Lipid Res. 2004;45:1375-1397.

3. Martín MG, Pfrieger F, Dotti CG. Cholesterol in brain disease: sometimes determinant and frequently implicated. EMBO Rep. 2014;15:1036-1052.

4. Porter FD, Herman GE. Malformation syndromes caused by disorders of cholesterol synthesis. J Lipid Res. 2011;52:6-34.

5. Nowaczyk MJM, Irons MB. Smith-Lemli-Opitz syndrome: phenotype, natural history, and epidemiology. Am J Med Genet C Semin Med Genet. 2012;262:250-262.

6. Blassberg R, Macrae Jl, Briscoe J, Jacob J. Reduced cholesterol levels impair Smoothened activation in Smith-Lemli-Opitz syndrome. Hum Mol Genet. 2016;25:693-705

7. Myers BR, Sever N, Chong YC, et al. Hedgehog pathway modulation by multiple lipid binding sites on the smoothened effector of signal response. Dev Cell. 2013;26:346-357.

8. Coman D, Vissers LELM, Riley LG, et al. Squalene synthase deficiency: clinical, biochemical, and molecular characterization of a defect in cholesterol biosynthesis. Am J Hum Genet. 2018;103:125-130.

9. Zhao L, Chen X-J, Zhu J, et al. Lanosterol reverses protein aggregation in cataracts. Nature. 2015;523:607-611.

10. Chen X, Liu L, Report C, Chen X, Liu L. Congenital cataract with LSS gene mutations: a new case report. J Pediatr Endocrinol Metab. 2017;30:1231-1235.

11. Romano M, Tafazzoli A, Mattern M, et al. Bi-allelic mutations in LSS encoding lanosterol synthase, cause autosomal-recessive hypotrichosis simplex. Am J Hum Genet. 2018;103:777-785.

12. Nes WD. Biosynthesis of cholesterol and other sterols. Chem Rev. 2011;111:6423-6451

13. Reza Sailani M, Jahanbani F, Nasiri J, et al. Association of AHSG with alopecia and mental retardation (APMR) syndrome. Hum Genet. 2017;136:287-296.

14. Pridmore C, Baraitser M, Brett EM. Alopecia, mental retardation, epilepsy and microcephaly in two cousins. Clin Dysmorphol. 1992;1:79-84.

15. Devriendt K, Van den Berghe H, Fryns JP. Alopecia-mental retardation syndrome associated with convulsions and hypergonadotropic hypogonadism. Clin Genet. 1996;49:6-9.

16. John P, Ali G, Chishti MS, Naqvi SMS, Leal SM, Ahmad W. Localization of a novel locus for alopecia with mental retardation syndrome to chromosome 3q26.33-q27.3. Hum Genet. 2006;118:665-667.

17. Wali A, John P, Gul A, et al. A novel locus for alopecia with mental retardation syndrome (APMR2) maps to chromosome 3q26.2-q26.31. Clin Genet. 2006;70:233-239.

18. Wali A, Ali G, John P, et al. Mapping of a gene for alopecia with mental retardation syndrome (APMR3) on chromosome 18q11.2-q12.2. Ann Hum Genet. 2007;71:570-577.
19. Tzschach A, Bozorgmehr B, Hadavi V, et al. Alopecia-mental retardation syndrome: clinical and molecular characterization of four patients. $\mathrm{Br} \mathrm{J}$ Dermatol. 2008;159:748-751.

20. Sobreira N, Schiettecatte F, Valle D, Hamosh A. GeneMatcher: a matching tool for connecting investigators with an interest in the same gene. Hum Mutat. 2015;36:928-930.

21. Soukarieh $O$, Gaildrat $P$, Hamieh $M$, et al. Exonic splicing mutations are more prevalent than currently estimated and can be predicted by using in silico tools. PLoS Genet. 2016;12:1-26.

22. Dzeletovic S, Breuer O, Lund E, Diczfalusy U. Determination of cholesterol oxidation products in human plasma by isotope dilution-mass spectrometry. Anal Biochem. 1995;225:73-80.

23. Chevy F, Humbert L, Wolf $C$. Sterol profiling of amniotic fluid: a routine method for the detection of distal cholesterol synthesis deficit. Prenat Diagn. 2005;25:1000-1006.

24. Kircher M, Witten DM, Jain P, O'Roak BJ, Cooper GM, Shendure J. A general framework for estimating the relative pathogenicity of human genetic variants. Nat Genet. 2014;46:310-315.

25. Lek M, Karczewski KJ, Minikel EV, et al. Analysis of protein-coding genetic variation in 60,706 humans. Nature. 2016;536:285-291.

26. Thoma R, Schulz-Gasch T, D'Arcy B, et al. Insight into steroid scaffold formation from the structure of human oxidosqualene cyclase. Nature. 2004:432:118-122.

27. Ruf A, Müller F, D'Arcy B, et al. The monotopic membrane protein human oxidosqualene cyclase is active as monomer. Biochem Biophys Res Commun. 2004;315:247-254.

28. Young M, Chen $\mathrm{H}$, Lalioti MD, Antonarakis SE. The human lanosterol synthase gene maps to chromosome 21q22.3. Hum Genet. 1996;97:620-624.

29. Huff MW, Telford DE. Lord of the rings-the mechanism for oxidosqualene:lanosterol cyclase becomes crystal clear. Trends Pharmacol Sci. 2005;26:335-340.

30. Roessler E, Mittaz L, Du Y, et al. Structure of the human lanosterol synthase gene and its analysis as a candidate for holoprosencephaly (HPE1). Hum Genet. 1999;105:489-495.

31. Mori M, Li G, Abe I, et al. Lanosterol synthase mutations cause cholesterol deficiency-associated cataracts in the Shumiya cataract rat. J Clin Invest. 2006;116:395-404.

32. Waterham HR, Koster J, Romeijn GJ, et al. Mutations in the $3 \beta$ Hydroxysterol $\Delta 24$-Reductase gene cause desmosterolosis, an autosomal recessive disorder of cholesterol biosynthesis. Am J Hum Genet. 2001;69:685-694

33. Andersson HC, Kratz L, Kelley R. Desmosterolosis presenting with multiple congenital anomalies and profound developmental delay. Am J Med Genet. 2002;113:315-319.

34. Mirza R, Hayasaka S, Takagishi Y, et al. DHCR24 gene knockout mice demonstrate lethal dermopathy with differentiation and maturation defects in the epidermis. J Invest Dermatol. 2006;126:638-647.

35. Fitzky BU, Moebius FF, Asaoka H, et al. 7-Dehydrocholesterol-dependent proteolysis of HMG-CoA reductase suppresses sterol biosynthesis in a mouse model of Smith-Lemli-Opitz/RSH syndrome. J Clin Invest. 2001;108:905-915.

36. Tozawa R, Ishibashi S, Osuga J, et al. Embryonic lethality and defective neural tube closure in mice lacking squalene synthase. J Biol Chem. 1999;274:30843-30848.

37. Panicker SP, Ganguly T, Consolo $M$, et al. Sterol intermediates of cholesterol biosynthesis inhibit hair growth and trigger an innate immune response in cicatricial alopecia. PLoS One. 2012;7:e38449

38. Goldenberg A, Chevy F, Bernard C, Wolf C, Cormier-Daire V. [Clinical characteristics and diagnosis of Smith-Lemli-Opitz syndrome and tentative phenotype-genotype correlation: report of 45 cases]. Arch Pediatr. 2003;10:4-10.

39. Goldenberg A, Wolf C, Chevy F, et al. Antenatal manifestations of SmithLemli-Opitz (RSH) syndrome: a retrospective survey of 30 cases. Am J Med Genet A. 2004;124A:423-426.

40. Fakheri RJ, Javitt NB. Autoregulation of cholesterol synthesis: physiologic and pathophysiologic consequences. Steroids. 2011;76:211-215. 
${ }^{1} \mathrm{CHU}$ de Nantes, Service de Génétique Médicale, Nantes, France. ${ }^{2}$ L'institut du thorax, INSERM, CNRS, UNIV Nantes, CHU de Nantes, Nantes, France. ${ }^{3}$ INSERM, UMR 1256 Nutrition-Genetics-Environmental Risk Exposure and Reference Centre of Inborn Metabolism Diseases, University of Lorraine and University Hospital Centre of Nancy (CHRU Nancy), Nancy, France. ${ }^{4}$ Department of Genetics, Rouen University Hospital, Normandy Centre for Genomic and Personalized Medicine, Rouen, France. ${ }^{5}$ Department of

Dermatology, University Hospital Center of Rouen, Rouen, France. ${ }^{6} \mathrm{CHU}$ de Nantes, Department of Dermatology, Nantes, France. ${ }^{7}$ Department of Medical Genetics, INSERM UMR 1163, Paris Descartes-Sorbonne Paris Cité University, IMAGINE Institute, Necker Enfants Malades Hospital, Paris, France. ${ }^{8}$ Section of Clinical and Metabolic Genetics, Department of Pediatrics, Hamad Medical Corporation, Doha, Qatar. ${ }^{9}$ Dermatology department, hôpital Brabois, Vandœuvre-Lès, Vandœuvre-lès-Nancy, France. ${ }^{10}$ Centre de Genetique et Centre de Reference Anomalies du Développement et Syndromes Malformatifs de l'Est, FHU-TRANSLAD, CHU Dijon, Dijon, France. ${ }^{11}$ UMR-Inserm 1231 GAD Team, Genetique des Anomalies du Développement, Université de Bourgogne FrancheComte, Dijon, France. ${ }^{12}$ Department of Pediatric Neurology and Developmental Medicine, University of Basel Children's Hospital (UKBB), Basel, Switzerland. ${ }^{13} \mathrm{CH}$ Auray-Vannes, Hôpital Bretagne Atlantique, Service de Pediatrie, Vannes, France. ${ }^{14}$ Ecole Pratique des Hautes Etudes, PSL Research University, Paris, France. ${ }^{15}$ Pediatric Neurology Unit, Cliniques Universitaires Saint-Luc, Universite Catholique de Louvain, Brussels, Belgium. ${ }^{16}$ Center of Autism and Department of Genetics, University Hospital Liege (CHU), Liège, Belgium. ${ }^{17}$ GeneDx, 207 Perry Parkway, Gaithersburg, MD, USA. ${ }^{18}$ Centre de Génétique, Hôpital Couple-Enfant, CHU de GrenobleAlpes, La Tronche, France. ${ }^{19}$ Centre de génétique humaine, Université de Franche-Comté, Besançon, France. ${ }^{20}$ Integrative and Cognitive Neurosciences Research Unit EA481, University of Franche-Comté, Besançon, France. ${ }^{21}$ Department Medical Genetic and Pathology, University Hospital of Basel (USB), Basel, Switzerland. ${ }^{22}$ Laboratory of Mass Spectrometry, INSERM ERL 1157, CNRS UMR 7203 LBM, Sorbonne Universités-UPMC, CHU Saint-Antoine, Paris, France 